\title{
Status and Perspectives of Liquid Argon Calorimeters
}

\author{
J. Engler
}

Institut für Kern- und Teillchenphysik

\section{Kernforschungszentrum Karlsruhe}




\section{KERNFORSCHUNGSZENTRUM KARLSRUHE}

Institut für Kern- und Teilchenphysik

$\mathrm{K} £ \mathrm{~K} \quad 3595$

Status and Perspectives of Liquid Argon Calorimeters

Joachim Engler

Kernforschungszentrum Karlsruhe GmbH, Karlsruhe 
Als Manuskript vervielfältigt

Für diesen Bericht behalten wir uns alle Rechte vor

Kernforschungszentrum Karlsruhe $\mathrm{GmbH}$

ISSN 0303-4003 
Status and Perspectives of Liquid Argon Calorimeters

ABSTRACT

The status of liquid argon calorimeters is reviewed, and experience obtained with these devices is described. Future perspectives of the liquid ionization chamber technique in calorimetry are also discussed.

Stand und Perspektiven von F:lüssig-Argon Kalorimetern

\section{ZUSAMMENFASSUNG}

Es wird eine Übersicht über den stand der Flüssigargon-Kalorimeter gegeben und über Erfahrungen mit diesen Nachweisgeräten berichtet. Ebenso werden die zukünftigen Möglichkeiten der Flüssigionisationskammer beim Einsatz in Großkalorimetern diskutiert.

Invited talk at the II. Pisa Conference on Advanced Detectors, June 3-7, 1983 


\section{INTRODUCTION}

Since the liquid argon ionization chamber was introduced into high energy calorinetry by willis and Radeka ${ }^{1)}$ about 10 years ago, several groups have applied this new detector technique. Starting from test devices many calorimeters have been developped up to large scale $4 \pi$ detectors in storage ring experiments. The first experiments using LAr calorimeters have already been completed, and it is therefore a good moment to review the experience and the performance obtained with this technique.

A compilation of all calorimeters used in experiments so far is given in Table 1. The first three have finished data taking, whereas the large detectors at the storage rings PEP and PETRA are continuing, and the MARK II calorimeter is approved for the SLC physics programme. Apart from a few test devices for hadron calorimetry (among which the famous Uranium calorimeter of C.W. Fabjan et al. ${ }^{8)}$ ), all devices built so far are electromagnetic calorimeters. So I will concentrate mainly on these.

TABLE 1: Electromagnetic Calorimeters in Experimental Detectors

\begin{tabular}{lclrrr}
\hline Lab. & Ref & Experiment & Pb Weight & LAr Volume No. channels \\
\hline CERN-ISR & 2 & Willis, Fabjan & $8 t$ & $1.500 \ell$ & 600 \\
CERN-SPS & 3 & Geneva-Lausanne & $2 \cdot t$ & $3.000 \ell$ & 220 \\
Fermilab & 4 & Rochester-FNAL & $2 t$ & $3.000 \ell$ & 800 \\
PEP & 5 & MARK II & $20 t$ & $8.000 \ell$ & 3.000 \\
PETRA & 6 & Tasso & $26 t$ & $10.000 \ell$ & 14.800 \\
PETRA & 7 & Cello & $36 t$ & $30.000 \ell$ & 11.000 \\
\hline
\end{tabular}

\section{ELECTROMAGNETIC CALORIMETERS}

In all calorimeters the sampling technique was used, i.e. the cascade of shower particles is absorbed in a stack of plates of dense material, and only part of the released ionization charge is detected by the liquid argon in the gaps between. The energy resolution of such a calorimeter is usually parametrized by the sun of three terms, which depend differently on the incoming particle energy $\mathrm{E}$ :

$$
(d \sigma / E)^{2}=(A / \sqrt{E})^{2}+(B / E)^{2}+C^{2}
$$


- Term A reproduces the sampling fluctuations caused by the undetected energy, which rests unseen in the inert absorber plates.

- Term B represents effects like electronic noise, electronic pick-up in a high intensity environment, and material in front of the active calorimeter. It contributes mainly at low energies below 1 GeV.

- Term $\mathrm{C}$ is due to wrong intercalibration between individual channels, mechanical inhomogeneities in the stack construction, malfunctioning channels, etc. It contributes at high energy, starting typically around $20 \mathrm{GeV}$.

The resolution achieved by different calorimeters varies, but typical paramẹters obtained are: $A \sim 7 \ldots$ 10\%, B $\sim 20 \%, C \sim 1 \ldots 2 \%$ with $E$ expressed in GeV.

Let us look in more detail into the sampling fluctuations, which usually represent the most relevant contribution. Table 2 gives a compilation of several calorimeters tested up to now and their energy resolution. The fixst nine devices use lead plates as converters, followed by two iron calorimeters, and the last calorimeter uses aluminium as absorber plates. It was recently tested by an IHEP/Pisa group at the Serpuchow accelerator.

For interpretation of the experimentally found resolution, the EGS Monte Carlo code of Ford and Nelson ${ }^{12}$ ) should be consultated. But for a quick estimate, a reasonably good estimate for the sampling fluctuations can be obtained following Rossi's approximation $\mathrm{B}^{13}$ ) of shower fluctuations in the following way: Due to the sampling method the total deposited energy $E$ is cut into $N$ small energy buckets $\Delta E$, where $\Delta E$ is the energy loss in one sampling step, i.e. in the inert and the adjacent active layer. Assuming the energy buckets to be statistically independent and applying Poisson statistic one obtains:

$$
\frac{d \sigma}{E} \sim \frac{1}{\sqrt{N}}=\sqrt{\frac{\Delta E}{E}}
$$

This rough estimate has to be refined for real calorimeters by taking into account two effects:

a) Experimentally the electrons and positrons in the cascade are detected in the active layer only above a certain threshold energy, which 
TABLE 2: Energy Resolution in LAr Test Calorimeters

\begin{tabular}{|c|c|c|c|c|c|c|c|c|}
\hline \multirow{2}{*}{ Authors, Group } & \multirow{2}{*}{ Ref. } & \multirow{2}{*}{$\mathrm{Cell}$} & \multirow{2}{*}{$1(\mathrm{~mm})$} & \multicolumn{2}{|c|}{ Sampling Step } & \multicolumn{2}{|c|}{$d \sigma / \sqrt{E}(8)$} & \multirow[b]{2}{*}{ Ratio } \\
\hline & & & & $x_{0}$ & $\Delta \mathrm{E}(\mathrm{MeV})$ & Test & Formula & \\
\hline Hitlin (SLAC) A & 9 & 1 & $\mathrm{~Pb} / 2 \mathrm{LAr}$ & .18 & 1.70 & 6.5 & 6.6 & .98 \\
\hline Hitlin (SLAC) B & 9 & 22 & $\mathrm{~Pb} / 4 \mathrm{LAr}$ & .42 & 3.66 & 9.5 & 9.6 & .99 \\
\hline $\begin{array}{c}\text { Asano (Tsukuba, } \\
\text { Tokyo) }\end{array}$ & 10 & 2 & $\mathrm{~Pb} / 2 \mathrm{LAr}$ & .37 & 2.98 & 9.6 & 8.7 & 1.10 \\
\hline $\begin{array}{l}\text { Mühlemann } \\
\qquad \text { (Geneva) A }\end{array}$ & 3 & 1 & $\mathrm{~Pb} / 4$ LAr & .23 & 2.76 & 8.0 & 8.4 & .95 \\
\hline $\begin{array}{lll}\| & \quad & B\end{array}$ & 3 & 2 & $\mathrm{~Pb} / 8 \mathrm{LAr}$ & .46 & 5.52 & 12.0 & 11.9 & 1.0 \\
\hline Tasso & 6 & 2 & $\mathrm{~Pb} / 5$ LAr & .39 & 3.62 & 10.0 & 9.6 & 1.04 \\
\hline Cello & 7 & 1.2 & $\mathrm{~Pb} / 3.6 \mathrm{LAr}$ & .24 & 2.3 & 9.0 & 7.7 & 1.17 \\
\hline Mark II & 5 & 2 & $\mathrm{~Pb} / 3 \mathrm{LAr}$ & .38 & 3.2 & 10.8 & 9.0 & 1.20 \\
\hline Nel:son (FNAL) & 4 & 2 & $\mathrm{~Pb} / 2$ LAr & .41 & 3.3 & 10.3 & 9.2 & 1.12 \\
\hline Fabjan,willis & 8 & 1.5 & $\mathrm{Fe} / 2 \mathrm{LAr}$ & .10 & 2.16 & 6.9 & 5.2 & 1.32 \\
\hline Asano & 10 & 2 & $\mathrm{Fe} / 2 \mathrm{LAX}$ & .13 & 2.74 & 6.1 & 5.9 & 1.03 \\
\hline $\begin{array}{r}\text { Sergiampietri } \\
\text { (IHEP) }\end{array}$ & 11 & 1 & $\mathrm{Al} / 3 \mathrm{LAR}$ & .033 & 1.07 & 3.6 & 3.4 & 1.06 \\
\hline
\end{tabular}

in shower theory is normally called the cut-off energy $E_{C}$. This reduces the total available energy by a fraction $F=F\left(E_{C} / \varepsilon\right)$, which depends on the ratio of $\mathrm{E}_{\mathrm{C}}$ to the critical energy $\varepsilon$ of the absorbing material. In our case of a LAr ionization chamber, a reasonable guess for $E_{C}$ is the energy loss of a minimum ionizing particle in a single LAr gap.

b) The lateral spread of the shower entails that the effective sampling thickness traversed by the particles increases by $1 /\langle\cos \theta\rangle$, where $<\cos \theta\rangle$ is the mean angle of all particles with the shower axis.

Taking into account these two effects, we can write for the sampling fluctuations

$$
\frac{d \sigma}{E} \sim \sqrt{\frac{\Delta E}{F \cdot\langle\cos \theta\rangle \cdot E}}
$$

A good parametrization for $F$ and $\left\langle\cos \theta>\right.$ has been given by $U$. Amaldi ${ }^{14}$ 
at the Uppsala Conference. Both factors are nearly unity for light elements like $A 1$ and become smaller than 1 for high $Z$ materials. The agreement of formula (3) with the experimental findings is very satisfactory as can be seen by comparing column 4 and 5 in Table 2 . The ratio scatters around unity.

The resolutions given in Table 2 were obtained under test conditions, that means only a limited number of channels was used and measurements were performed for relatively short periods of beam time. Meanwhile the large detectors have accumulated data for several years, and we can compare the test results with the resolutions obtained under long term experimental conditions. This is demonstrated in Table 3 , where in co-

TABLE 3: Performance of LAr Calorimeters under Experimental Cóniditions

\begin{tabular}{|c|c|c|c|c|}
\hline \multirow{2}{*}{ Detector } & \multirow{2}{*}{\multicolumn{2}{|c|}{$\begin{array}{l}\text { Calibration Method } \\
\text { (Energy Range) }\end{array}$}} & \multicolumn{2}{|c|}{$d \sigma / \sqrt{E}\left(\frac{o}{b}\right)$} \\
\hline & & & Test & Experiment \\
\hline $\begin{array}{l}\text { FNAL Photon Cal. } \\
\text { (Nelson et al.) }\end{array}$ & $\mathrm{e}^{+}$from $\mathrm{ke}_{3}$ & $(15.70 \mathrm{GeV})$ & 10.3 & 14 \\
\hline $\begin{array}{l}\text { Geneva Photon Cal. } \\
\text { (Mühlemann) }\end{array}$ & $e^{-}$beam & $(6 \mathrm{GeV})$ & 12 & 15 \\
\hline $\begin{array}{l}\text { ISR Photon Cal. } \\
\text { (Cobb et al.) }\end{array}$ & $\mathrm{e}^{-}$beam & $(.75-4 \mathrm{GeV})$ & 8 & 10 \\
\hline \multicolumn{5}{|l|}{ PETRA Detector } \\
\hline TASSO & Bhabha & $(17 \mathrm{GeV})$ & 10 & 16 \\
\hline CELLO & Bhabha & $(17 \mathrm{GeV})$ & 9 & $11+1.8 \sqrt{E}$ \\
\hline \multicolumn{5}{|l|}{ PEP Detector } \\
\hline MARK II & Bhabha & $(2 \mathrm{GeV})$ & 10.8 & 11.5 \\
\hline
\end{tabular}

lumn 3 and 4 test results are given together with the final experimental findings. The method of calibration used during the experiment is given in column 2. One observes that usually the energy resolution becomes worse by two to three per cent. This deterioration is attributed mostly to non-responding channels and to uncertainties in the intercalibration system.

A further deterioration shows up for complex event pattern or in a high intensity environment, where problems of overlapping showers have to be 
encountered. The effect depends very much on the individual experimental condition and on the layout of the longitudinal and lateral segmentation in the calorimeter, i.e. the strip or pad structure. How important this effect can be, is demonstrated in Fig. 1, which shows a result from the FNAL photon calorimeter ${ }^{4}$. In the decay of $\mathrm{K}^{+} \rightarrow \mathrm{e}^{+} \pi^{0} \mathrm{v}$ three showers have to be disentangled. The energy resolution found for the positrons, which are also magnetically analyzed, is worse by roughly a factor 1.5 as compared to a single electron calibration beam.

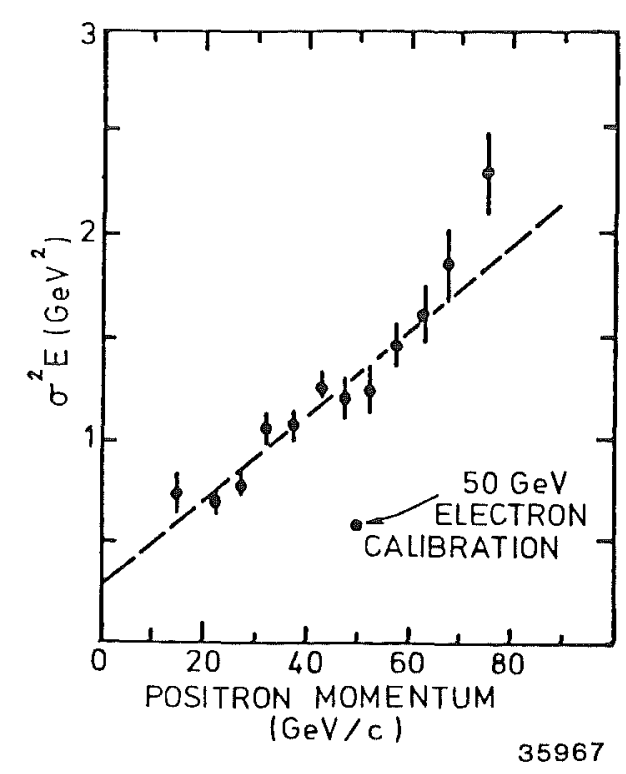

Fig. 1:

Energy Resolution for Positrons from $\mathrm{ke}_{3}$ Decay as obtained in the FNAL Calorimeter (Ref. 4)

\section{HADRON CALORIMETERS}

Up to now only test calorimeters were built, and LAr hadron calorimeters have not been used in experimental detectors. This might change in the future, because of the nice property of a liquid ionization chamber being insensitive to radiation damage. I will not comment on the wellknown Uranium calorimeter of Willis and Fabjan ${ }^{8)}$ but restrict myself to mention two iron calorimeters. Both used thin plates, the CERN calorimeter $1.5 \mathrm{~mm}$ Fe and the FNAL calorimeter of A.L. Sessoms et al. ${ }^{15)} 3 \mathrm{~mm}$ Fe. The outcome of the combined data is that the energy resolution is not consistent with a simple $\sigma / E \sim A / \sqrt{E}$ dependence. This was confirmed by a Monte Carlo calculation of T.A. Gabriel et al. ${ }^{16)}$, performed with the HETC Oak Ridge code up to $250 \mathrm{GeV}$ hadron energy. The energy resolution of the experimental data can be parametrized as follows (up to $38 \mathrm{GeV}$ ): 


$$
\mathrm{d \sigma} / \mathrm{E}=8.5 \%+28 \% / \sqrt{\mathrm{E}} .
$$

Including the M.C. points (up to $250 \mathrm{GeV}$ ) one rather obtains

$$
\mathrm{do} / \mathrm{E}=5 \%+33 \% / \sqrt{\mathrm{E}} \text {. }
$$

\section{EXPERIENCE WITH LAT CALORIMETERS}

\subsection{Cryostat and Cryogenics}

Here we have a typical threshold effect. Groups which started with this new technique had to learn, but once familiar with the system all groups unanimously report of having encountered no long term difficulties. As an example, the CELLO calorimeter had to be warmed up and moved out of the beam. All stacks were completely rewired and cooled down again without problems after one year. When the LAr was filled back again, the same electrical pulse height was observed as compared to one year ago.

\section{2 LAr Purity}

None of the groups has complained about any problem with oxygen contamination. The purity is reported to stay always below $1 \mathrm{ppm}$ for a merely infinite long time. However, several groups have reported about charges of liquid argon delivered from industry, which gave all of a sudden a reduced signal output, probably due to an unknown impurity in the liquid. Recently TASSO observed such a decrease of $30-50 \%$ when they changed the delivering company for liquid argon. Whether the argon is contaiminated during fabrication or during transportation is an open question. The best remedy is to check the arriving liquid in a small test chamber.

\subsection{High Voltage and Electronics}

Up to $10 \mathrm{kVolt} / \mathrm{cm}$ usually no problems are encountered, provided the liquid is perfectly clean (probably passed through filters) and all the materials used in the cryostat, as e.g. the electrode surfaces, are resistant to shocks during the cool-down phase. Lost channels due to high voltage short circuits can be kept at a very low level. The FNAL photon calorimeter reports a loss of less than $1 \%$, TASSO and CELIO have typically $0.5 \%$.

The calibration is normally stable within less than $1 \%$ for many months, as is reported by the experimental groups. 
Special care, of course, is needed when seeting up and maintaining the sensitive low noise amplifier system. Problems with ringing and pickup is a function of the conscientiousness of the responsible physicists. Both effects are especially troublesome in a trigger system, but can be mastered. At CELLO a single photon trigger operates with a threshold of $2 \mathrm{GeV}$ photon energy at a trigger rate of about $0.5 / \mathrm{sec}$.

\section{NEW DEVELOPMENTS}

High counting rates are a certain limitation for liquid ionization chambers due to the slow drifttime of $\sim 200 \mathrm{nsec} / \mathrm{mm}$. Muhlemann and Tavano ${ }^{17)}$ have shown that with $1 \%$ admixture of methane the drifttime can be reduced to $80 \mathrm{nsec} / \mathrm{mm}$ without losing in pulse height for the signal of a minimum ionizing particle. But up to now, methane was not used in any of the large detectors.

How well, on the other hand, a timing can be achieved has been demonstrated by the FNAL photon calorimeter ${ }^{4}$. A resolution of $\sigma=6 \mathrm{nsec}$ could be obtained as shown in Fig. 2.

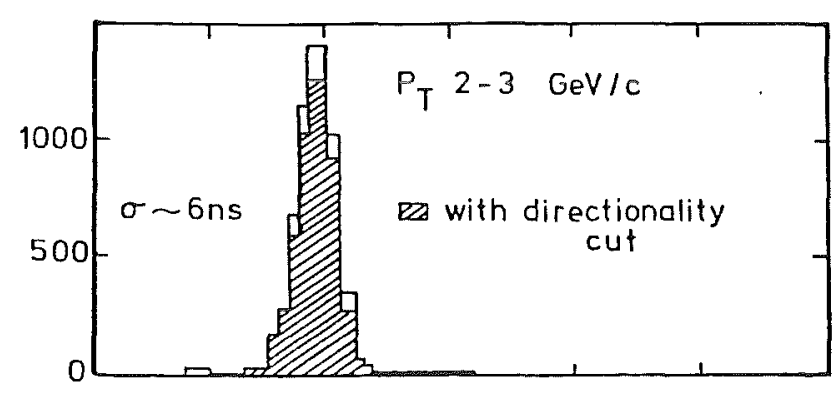

Fig. 2:

Time Resolution in the FNAL Calorimeter for Photons (Ref. 4)

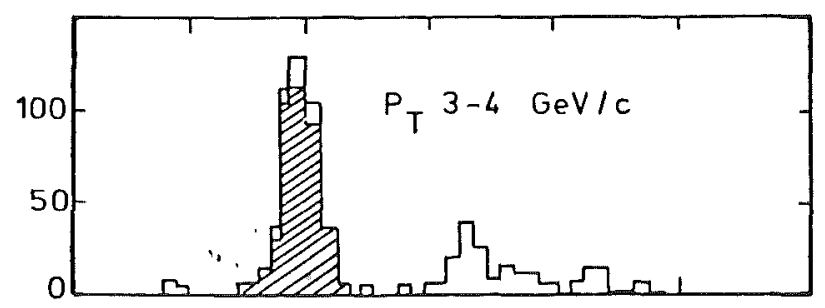

PHOTON TIME (ns)

35966

An interesting possibility of read-out was investigated at orsay by $D$. Fournier and coworkers ${ }^{18}$ ). They tried to read out the charge on both electrodes, the high voltage as well as the ground side. In this way the same signal can be obtained on different geometrical patterns, e.g. 
strips on one and pads on the other electrode or strips with different orientations. A very good pulse height correlation was found, which in practice is limited only by the electronic noise, and this strong correlation helps to resolve ambiguities in pattern recognition.

\section{FUTURE PERSPECTIVES}

There are three new proposals to use LAr calorimeters in experiments: At CERN the CP violation experiment for neutral decay of $K_{L, S}^{\circ}$, at serpuchow the 300 ton neutrino detector, and at TRISTAN the venus detector, which intends to use LAr calorimeters in the end caps. For LEP detectors, LAr calorimeters have been disregarded, mostly because of space problems inside magnetic coils.

At several places interesting developments are going on to use the liquid argon as homogeneous detectors with application mainly in neutrino physics or for rare decay experiments.

\subsection{Homogeneous Calorimeters}

In a homogeneous calorimeter the electrodes have to be nearly massless. This can be realized by large gap distances which means long drifttimes. H. Chen and coworkers ${ }^{19)}$ at Irvine have investigated in detail the corresponding problems of purification down to the ppb level oxygen equivalent. They obtained an attentuation length of $\lambda \sim 55 \mathrm{~cm}$ at a reasonably low electrical field of $1.6 \mathrm{kV} / \mathrm{cm}$. Thus, if care is taken, a liquid argon time projection chamber seems to be feasible.

Avoiding the problem of electron attachment, the Pisa group has run the IHEP/Pisa calorimeter not in the usual charge integration mode but in a current mode 20 ). They use only the first current after the particle has passed through the calorimeter and do not collect the later arriving ionization chàrges. The gap distance used was $28 \mathrm{~mm}$ between $1 \mathrm{~mm}$ aluminium electrodes. For $36 \mathrm{GeV}$ electrons an energy resolution of $\sigma / \mathrm{E}=0.85 \%$ was measured and with the beam spread unfolded $\sigma / E=0.78 \%$. This is, as an absolute value, the best resolution ever obtained in a liquid argon calorimeter. 


\subsection{Scintillation Light}

An interesting extension for calorimetry is to use the scintillation light in addition to charge collection. Since long the noble gases are known to be perfect scintillators (see for instance T. Doke ${ }^{21}$ ). Unfortunately, the light is in the far ultra-violet, for argon at $125 \mathrm{~nm}$. But it can be efficiently used, e.g. for trigger purposes.

A 50 tons liquid argon ball acting as a homogeneous calorimeter is under investigation to study the rare decay of $\mathrm{K}^{+} \rightarrow \pi^{+} \nu$ by $\mathrm{M}$. Ferro-Luzzi and coworkers $^{22}$ at CERN. Both the ionization charge and the scintillation light are used. the latter serving to trigger on the successive decay of the pion. A similar detector is under study for neutrino physics at the Neutron Spallation Source at Rutherford Laboratory ${ }^{23)}$.

\subsection{Argon Bubble Chambers}

The feasibility of a LAr bubble chamber is investigated at CERN by Harigel and coworkers ${ }^{24}$ ) and also at FNAL to be used in neutrino physics. Transparent wire electrodes are supposed to collect the charge operating the total argon volume as a calorimeter. In addition, the scintillation light is used to trigger the chamber optics.

A solid argon and neon calorimeter has been developped at Ecole Polytechnique by Brisson et al. ${ }^{25)}$ and was successfully tested in a low intensitiy beam at CERN. It is intended to be used at the downstream side of $\mathrm{BEBC}$ filled with $\mathrm{H}_{2}$ to detect electrons and photons in neutrino events events. No problems with pulse height reduction due to positive ion shielding were observed for particle fluxes below 4 particles/ $\mathrm{cm}^{2} \mathrm{sec}$.

\subsection{Warm Liquids}

Often the question is raised why only argon can be used in a liquid ionization chamber. The problem is electron attachment: Most liquids are electrical polar. An electron liberated in the ionization process is immediately caught by a molecule forming a negatively charged molecule, which moves too slowly and is thereby lost for charge collection. Only the noble gases $\mathrm{Ar}, \mathrm{Kr}$ and $\mathrm{Xe}$ are non-polar and all the ionization charge can be collected. Among the molecules methane is reasonably polar due to its tetrahedral structure, but unfortunately it is also cryogenic. There exist room temperature liquids with a super methane structure, na- 
mely a four valent element in the centre of a tetrahydron and four methyl radicals around it: $\mathrm{x}\left(\mathrm{CH}_{3}\right)_{4}$. Among the existing molecules with $\mathrm{x}=$ $\mathrm{C}, \mathrm{Si}, \mathrm{Ge}, \mathrm{Sn}$, and $\mathrm{Pb}$, the liquids with $\mathrm{Si}$ and $\mathrm{Sn}$ seem to be the most promising ones from technical point of view. At Karlsruhe a test chamber with tetramethyl-silane is operating. At a voltage of $20 \mathrm{kV} / \mathrm{cm}$ a charge output for cosmic muons of $35 \%$ as compared to LAr has been obtained. We have to wait for the future to see whether there will open up new developments along these lines. Further improvements might show up in this new, but by now already 10 years old technique.

\section{REFERENCES}

1) W.J. Willis and V. Radeka, NIM 120 (1974) 221

2) J.H. Cobb et al., NIM 158 (1979) 93

3) A. Delfosse et al., NIM 156 (1978) 425 and P. Muhlemann, Preprint Université de Genève UGVA-DPNC 1983/03-111

4) C. Nelson et al., Preprint FERMILAB-Pub-82/41-EXP, submitted to NIM

5) G.S. Abrams et al., IEEE Transactions on Nuclear Science NS-27 (1980) 59

6) A. Ladage, SLAC-Report 250 (198z) 180

7) H.-J. Behrend et al., Physica Scripta 23 (1981) 610

8) C.W. Fabjan et al., NIM 141 (1977) 61

9) D. Hitlin et al., NIM $13 \overline{7}$ (1976) 225

10) Y. Asano et al., NIM $1 \overline{74}$ (1980) 357

11) C. Cerri et al., Preprint 1982 INFN, Sezione di Pisa, submitted to NIM

12) P.L. Ford and W.R. Nelson, SLAC-Report 210 (1978)

13) B. Rossi, High Energy Particles, Prentice-Hall, New York (1952)

14) U. Amaldi, Physica Scripta 23 (1981) 409

15) A.L. Sessoms et al., NIM $\frac{16 \overline{1}}{195}(1979) 371$

16) T.A. Gabriel et al., NIM $\overline{195}$ (1982) 461

17) P. Muhlemann and R. Tavano, NIM 166 (1979) 583

18) R.L. Chase et al., Orsay Preprint 1983, submitted to NIM

19) H.H. Chen and P.J. Doe, IEEE Transactions on Nuclear Science NS-28 (1980 454 and NS-29 (1982) 354

20) C. Cerri et al., Pisa Internal Report unpublished (1983)

21) T. Doke, Portugaliae Phys. 12 (1981) 9

22) M. Ferro-Luezi et al., unpublished report 1982

23) J. Engler, unpublished report 1982

24) J.C. Berset et al., NIM 203 (1982) 133

G. Harigel et al., CERN Preprint EP/83-47, submitted to NIM

25) V. Brisson et al., Preprint Ecole Polytechnique (1983), submitted to NIM 